\title{
ANALYTICITY OF THERMO-ELASTIC SEMIGROUPS WITH COUPLED HINGED/NEUMANN B.C.
}

\author{
IRENA LASIECKA AND ROBERTO TRIGGIANI
}

\begin{abstract}
We consider a thermo-elastic plate system where the elastic equation does not account for rotational forces. We select the case of hinged mechanical B.C. and Neumann thermal B.C., which are coupled on the boundary. We show that the corresponding s.c. contraction semigroup (on a natural energy space) is analytic and, hence, uniformly stable. Because of the boundary (high) coupling, this case of B.C. is not contained in, and is more challenging than, recent known cases of the literature [L-R.1], [L-L.1], [L-T.1].
\end{abstract}

\section{Introduction. Problem statement. Main result}

Dynamics. Let $\Omega$ be a two-dimensional bounded domain with smooth boundary $\Gamma$. On $\Omega$ we consider a thermo-elastic plate problem in the displacement $w$ and in the temperature $\theta$, where the elastic equation does not account for rotational forces. Moreover, in this paper, we focus on the case of coupled B.C. which arise with hinged mechanical B.C. and Neumann (Robin) thermal B.C. (see literature below). The model, once stripped from lowerorder terms and with (inessential) constants normalized to 1 , is as follows

1991 Mathematics Subject Classification. Primary: 35Q72; Secondary: 47D06, 73B30, $73 \mathrm{~K} 10$.

Key words and phrases. Thermo-elastic semigroups, coupled/hinged Neumann boundary conditions.

Research partially supported by the National Science Foundation under Grand DMS9504822 and by the Army Research Office under Grant DAAH04-96-1-0059. Presented at workshop on "Deterministic and stochastic evolution equations," Scuola Normale Superiore, Pisa, Italy, July 1997; IFIP Conference, Detroit, July 1997; MMAR'97, Miedzyzdroje, Poland, August 1997; Conference on Differential Equations, Vanderbilt U., November 1997.

Received: January 3, 1998. 
[Lag.1]:

$$
\begin{cases}w_{t t}+\Delta^{2} w+\Delta \theta=0 & \text { in }(0, T] \times \Omega=Q ; \\ \theta_{t}-\Delta \theta-\Delta w_{t}=0 & \text { in } Q ; \\ w(0, \cdot)=w_{0} ; w_{t}(0, \cdot)=w_{1} ; \theta(0, \cdot)=\theta_{0} & \text { in } \Omega ; \\ w \equiv 0 ; \Delta w+(1-\mu) B_{1} w+\theta=0 & \text { in }(0, T] \times \Gamma \equiv \Sigma ; \\ \frac{\partial \theta}{\partial \nu}+b \theta=0 & \text { on } \Sigma ; \\ B_{1} w=-c(x) \frac{\partial w}{\partial \nu} & \text { on } \Sigma .\end{cases}
$$

The assumption that the boundary $\Gamma$ be smooth means that, in particular, the mean curvature $c(\cdot) \in L_{\infty}(\Gamma)$.

Abstract setting. First, we let $\mathcal{A}$ be the following positive, self-adjoint operator on $L_{2}(\Omega)$ :

$$
\begin{aligned}
& \mathcal{A} h=\Delta^{2} h \\
& \mathcal{D}(\mathcal{A})=\left\{h \in H^{4}(\Omega) \cap H_{0}^{1}(\Omega): \Delta h+(1-\mu) B_{1} h=0 \text { on } \Gamma\right\} .
\end{aligned}
$$

Next, we introduce the positive self-adjoint operators $\mathcal{A}_{D}$ and $\mathcal{A}_{N}$, respectively:

(1.3) $\mathcal{A}_{D} h=-\Delta h ; \mathcal{D}\left(\mathcal{A}_{D}\right)=H^{2}(\Omega) \cap H_{0}^{1}(\Omega)$,

(1.4) $\mathcal{A}_{N} h=-\Delta h ; \mathcal{D}\left(\mathcal{A}_{N}\right)=\left\{h \in H^{2}(\Omega):\left[\frac{\partial h}{\partial \nu}+b h\right]_{\Gamma}=0\right\}, b>0$.

We have [Gr.1],

$$
\mathcal{D}\left(\mathcal{A}^{\frac{1}{2}}\right)=H^{2}(\Omega) \cap H_{0}^{1}(\Omega)=\mathcal{D}\left(\mathcal{A}_{D}\right) \text { (equivalent norms). }
$$

Accordingly, we introduce the following space (equivalent norms),

$$
\begin{aligned}
Y & =\left[H^{2}(\Omega) \cap H_{0}^{1}(\Omega)\right] \times L_{2}(\Omega) \times L_{2}(\Omega)=\mathcal{D}\left(\mathcal{A}^{\frac{1}{2}}\right) \times L_{2}(\Omega) \times L_{2}(\Omega) \\
& =\mathcal{D}\left(\mathcal{A}_{D}\right) \times L_{2}(\Omega) \times L_{2}(\Omega),
\end{aligned}
$$

and the following Green map $G$, defined by

$$
h=G g \Leftrightarrow\left\{\begin{aligned}
\Delta^{2} h & =0 \text { in } \Omega ; \\
\left.h\right|_{\Gamma} & =0 ;\left.\left(\Delta+(1-\mu) B_{1}\right) h\right|_{\Gamma}=g .
\end{aligned}\right.
$$

Elliptic regularity [L-M.1; p. 188-189] gives:

$$
\begin{aligned}
G: \text { continuous } L_{2}(\Gamma) \rightarrow H^{\frac{5}{2}}(\Omega) \cap H_{0}^{1}(\Omega) & \subset H^{\frac{5}{2}-4 \epsilon}(\Omega) \cap H_{0}^{1}(\Omega) \\
& =\mathcal{D}\left(\mathcal{A}^{\frac{5}{8}-\epsilon}\right), \quad \epsilon>0
\end{aligned}
$$

and

$$
\mathcal{A}^{\frac{5}{8}-\epsilon} G: \text { continuous } L_{2}(\Gamma) \rightarrow L_{2}(\Omega),
$$


where the identification in (1.8a) follows from [G.1]. By Green's second theorem, one readily obtains (see e.g., [L-T.4, Chapter 3, Section 12] for details)

$$
G^{*} \mathcal{A} f=\frac{\partial f}{\partial \nu}, \quad f \in \mathcal{D}(\mathcal{A})
$$

where $(G g, y)_{L_{2}(\Omega)}=\left(g, G^{*} y\right)_{L_{2}(\Gamma)}, g \in L_{2}(\Gamma), y \in L_{2}(\Omega)$. Using the definitions of $G, \mathcal{A}, \mathcal{A}_{D}, \mathcal{A}_{N}$ in (1.8), (1.2)-(1.4), we rewrite problem (1.1), as usual, as the following second-order abstract system ([L-T.4, Chapter 3, Section 12]):

$$
\left\{\begin{aligned}
w_{t t}+\mathcal{A} w+\mathcal{A} G\left(\left.\theta\right|_{\Gamma}\right)-\mathcal{A}_{N} \theta & =0 \text { in }[\mathcal{D}(\mathcal{A})]^{\prime} \\
\theta_{t}+\mathcal{A}_{N} \theta+\mathcal{A}_{D} w_{t} & =0 .
\end{aligned}\right.
$$

Setting $y=\left[w, w_{t}, \theta\right]$, we then rewrite the above second-order system as the first-order equation

$$
\dot{y}=A y, A=\left[\begin{array}{ccc}
0 & I & 0 \\
-\mathcal{A} & 0 & \mathcal{A}_{N}-\mathcal{A} G\left(\left.\cdot\right|_{\Gamma}\right) \\
0 & -\mathcal{A}_{D} & -\mathcal{A}_{N}
\end{array}\right]: Y \supset \mathcal{D}(A) \rightarrow Y
$$

to be interpreted in the sense that

$$
A=\left[\begin{array}{c}
w_{1} \\
w_{2} \\
\theta
\end{array}\right]=\left[\begin{array}{c}
w_{2} \\
-\mathcal{A}\left[G\left(\left.\theta\right|_{\Gamma}\right)+w_{1}\right]+\mathcal{A}_{N} \theta \\
-\mathcal{A}_{D} w_{2}-\mathcal{A}_{N} \theta
\end{array}\right],\left[\begin{array}{c}
w_{1} \\
w_{2} \\
\theta
\end{array}\right] \in \mathcal{D}(A),
$$

where, recalling $Y$ in (1.6), we obtain from (1.13),

$$
\begin{array}{r}
\mathcal{D}(A)=\left\{w_{1} \in \mathcal{D}\left(\mathcal{A}_{D}\right)=\mathcal{D}\left(\mathcal{A}^{\frac{1}{2}}\right) ; w_{2} \in \mathcal{D}\left(\mathcal{A}_{D}\right) ; \theta \in \mathcal{D}\left(\mathcal{A}_{N}\right) ;\right. \\
\left.w_{1}+G\left(\left.\theta\right|_{\Gamma}\right) \in \mathcal{D}(\mathcal{A})\right\} .
\end{array}
$$

Semigroup generation. The following result can be proved by standard methods: part (i) via the Lumer-Phillips Theorem; part (ii) by direct computation; see e.g. [L-T.4, Chapter 3, Section 12] for details.

Proposition 1.1. (i) The operator $A$ in (1.13), (1.14) is densely defined, dissipative, in fact maximal dissipative, and thus generates a s.c. contraction semigroup: $\left[w_{1}, w_{2}, \theta_{0}\right] \in Y \rightarrow e^{A t}\left[w_{1}, w_{2}, \theta_{0}\right]=\left[w(t), w_{t}(t), \theta(t)\right]$ on $Y$.

(ii) The operator $A$ has compact (inverse $A^{-1}$, explicitly given in [L-T.4, Chapter 3, Section 12], hence) resolvent on $Y$, and there is no spectrum $(=$ point spectrum) of $A$ on the closed right half-plane $\{\lambda: \operatorname{Re} \lambda \geq 0\}$.

Analyticity of $e^{A t}$. The goal of this paper is to prove the following.

Theorem 1.2. The s.c. contraction semigroup $e^{A t}$ of Proposition 1.1 is, moreover, analytic on $Y, t>0$. 
Uniform stability of $e^{A t}$. Once Theorem 1.2 is established, it then follows via Proposition 1.1(ii) that the s.c. contraction, analytic semigroup $e^{A t}$ is also uniformly stable in $\mathcal{L}(Y)$.

Corollary 1.3. There exist constants $M \geq 1$ and $\sigma>0$, such that $\left\|e^{A t}\right\|_{\mathcal{L}(Y)} \leq M e^{-\sigma t}, t \geq 0$.

Literature. The elastic equation (1.1a) is of Euler-Bernoulli type and thus it does not account for rotational terms. At first, thermo-elastic plate equations with (Kirchoff-type) or without (Euler-Bernoulli-type) rotational terms were the object of several studies showing asymptotic exponential stability of their solutions. See a detailed literature overview with a comprehensive list of references in [Lag.1], [Las.1], [L-R.1], [L-L.1], [L-T.1]. This means that heat dissipation alone is sufficiently strong to induce exponential energy decay.

Elastic model with no rotational term. Here, however, it was only recently that a much stronger and more desirable result was established in [L-R.1], at least for one demanding set of B.C., via a technical ad hoc proof: that in the case of clamped B.C. for $w /$ Dirichlet B.C. for $\theta$, the associated s.c. contraction semigroup is, in fact, analytic. [Once analyticity is established, it is not difficult to infer that the semigroup is also uniformly stable, by excluding the possibility that the generator has spectrum on the imaginary axis]. It is plainly desirable to have an abstract setting and an abstract proof of analyticity, which covers and encompasses at least several sets of physical B.C. Such goal is achieved in both the recent papers [L-L.1] and in [L-T.1] (see also [L-T.4, Chapter 3; Appendix F] and [T.1]). The proof in [L-L.1] is by a contradiction argument: it assumes that the well-known characterization of analyticity based on the resolvent of the generator is violated and gets a contradiction. By contrast, two direct proofs of analyticity for an abstract thermo-elastic model are given in [L-T.1]. Though technically and conceptually very different, these two proofs of [L-T.1] have in common the following basic idea: to fall into the setting of [C-T.1-2], and use the analyticity of the $2 \times 2$ structurally damped matrix operator as the main 'driver,' which is responsibile for carrying the analyticity of the heat component onto the mechanical component through the coupling. Moreover, [L-T.1] presents several P.D.E. thermo-elastic plate examples which recover, in particular: (i) the case of clamped B.C. in $w /$ Dirichlet B.C. for $\theta$ treated ad hoc in [L-R.1]; (ii) the case of physical hinged B.C./Dirichlet B.C. for $\theta$ treated ad hoc in [T.1]; (iii) the case of partially clamped/partially hinged B.C. in $w$ and Dirichlet B.C. in $\theta$, which is the only thermo-elastic illustration for analyticity given in [L-L.1]. In adition, [L-T.1] offers a few more examples: clamped/Neumann B.C.; and a damped free B.C. It should be noted that both the abstract operator theoretic thermo-elastic model of [L-T.1], [L-T.4, Chapter 3; Appendix F] and the variational, differently conceived, abstract model of [L-L.1] do not cover the case of coupled B.C. such as they occur in $(1.1 \mathrm{~d}-\mathrm{e})$. Moreover, their abstract, operator proofs cannot be adjusted 
to include a problem such as (1.1), which-because of the high coupling in the B.C. between $w$ and $\theta$, represents an additional serious level of difficulty over [L-L.1], [L-T.1] when it comes to showing analyticity. By contrast, the proof of analyticity of the present paper requires P.D.E. methods and trace estimates. An even much more demanding case - the case of free B.C.-will be handled separately, again by ad hoc, more elaborate, P.D.E. methods and trace estimates [L-T.5].

Elastic model with rotational terms. Here the structure of the corresponding s.c. semigroup is quite different; and is more akin to the stucture of a s.c. uniformly stable group (hence not analytic, not differentiable, not even continuous in the uniform topology for $t>0$, not compact); see [Cg-T.1], [L-T.2]; see also [H-P-L.1] for a different thermo-elastic wave (rather than plate) model, and [T-Z.1] for the specific case of a thermo-elastic plate with clamped/Dirichlet B.C.

The focus of this paper is Theorem 1.2 on analyticity. Results in Section 1 leading to this are standard and hence simply stated.

\section{Proof of Theorem 1.2}

2.1. General strategy and preliminaries. General strategy. With reference to the space $Y$ in (1.6), let $f_{0} \in Y$ be arbitrary

$$
\left\{\begin{array}{r}
f_{0}=\left[u_{0}, v_{0}, \theta_{0}\right] \in Y \equiv \mathcal{D}\left(\mathcal{A}^{\frac{1}{2}}\right) \times L_{2}(\Omega) \times L_{2}(\Omega), \\
\mathcal{D}\left(\mathcal{A}^{\frac{1}{2}}\right)=\mathcal{D}\left(\mathcal{A}_{D}\right)=H^{2}(\Omega) \cap H_{0}^{1}(\Omega) \text { (equivalent norms). }
\end{array}\right.
$$

With reference to the operator $A$ in (1.12)-(1.14), let $\omega$ be real, $\omega \in \mathbb{R}$, and define

$$
y(\omega)=[u(\omega), v(\omega), \theta(\omega)]=[i \omega I-A]^{-1} f_{0}=R(i \omega, A) f_{0} \in \mathcal{D}(A),
$$

where the resolvent of $A$ is well-defined on the imaginary axis, see Proposition 1.1(ii).

Our goal is to show that the following uniform estimate holds true: there exists a constant $C>0$ such that for all $\omega \in \mathbb{R}$, with say $|\omega| \geq 1$,

$$
\left\|\left[\begin{array}{l}
u(\omega) \\
v(\omega) \\
\theta(\omega)
\end{array}\right]\right\|_{Y}=\|y(\omega)\|_{Y}=\left\|R(i \omega, A) f_{0}\right\|_{Y} \leq \frac{C}{|\omega|}\left\|f_{0}\right\|_{Y} .
$$

Once estimate (2.1.3) has been established for the generator $A$ of the s.c. contraction semigroup $e^{A t}$ asserted by Proposition 1.1, we can invoke a known result e.g. [L-T.4, Chapter 3, Theorem E.3 of Appendix E] and obtain that the s.c. semigroup $e^{A t}$ is, in fact, analytic on $Y, t>0$. In order to establish estimate (2.1.3) - and hence prove Theorem 1.2-we shall pursue the following strategy which consists in proving the following three simultaneous estimates for the components of $y(\omega)$ in (2.1.2): for all $\epsilon>0$ there exists a constant $C_{\epsilon}>0$, such that for all $\omega \in \mathbb{R}$, with $|\omega|>1$, the vector 
$y(\omega)=[u(\omega), v(\omega), \theta(\omega)]$ in $(2.1 .2)$ satisfies

$$
\left\{\begin{aligned}
\|u(\omega)\|_{H^{2}(\Omega)}^{2} & \leq \epsilon\|y(\omega)\|_{Y}^{2}+C_{\epsilon}\left\|\frac{f_{0}}{\omega}\right\|_{Y}^{2} ; \\
\|v(\omega)\|_{L_{2}(\Omega)}^{2} & \leq \epsilon\|y(\omega)\|_{Y}^{2}+C_{\epsilon}\left\|\frac{f_{0}}{\omega}\right\|_{Y}^{2} ; \\
\|\theta(\omega)\|_{L_{2}(\Omega)}^{2} & \leq \epsilon\|y(\omega)\|_{Y}^{2}+C_{\epsilon}\left\|\frac{f_{0}}{\omega}\right\|_{Y}^{2}
\end{aligned}\right.
$$

Hereafter, we drop from $y=[u, v, \theta]$ the explicit dependence on $\omega$. Estimates (2.1.4)-(2.1.6) are proved below, in Proposition 2.4.1, Eqn. (2.4.1); Proposition 2.6.1, Eqn. (2.6.1); and Proposition 2.7.1, Eqn. (2.7.1), respectively. Clearly, summing up estimates (2.1.4) through (2.1.6) (once established) yields the final desired estimate $(2.1 .3)$ with constant $C=\left(3 C_{\epsilon} / 1-3 \epsilon\right)^{\frac{1}{2}}$.

Preliminaries. By (1.13), we obtain explicitly from (2.1.2),

$$
(i \omega I-A)\left[\begin{array}{l}
u \\
v \\
\theta
\end{array}\right]=\left[\begin{array}{c}
i \omega u-v \\
i \omega v+\mathcal{A}\left[u+G\left(\left.\theta\right|_{\Gamma}\right)\right]-\mathcal{A}_{N} \theta \\
i \omega \theta+\mathcal{A}_{D} v+\mathcal{A}_{N} \theta
\end{array}\right]=\left[\begin{array}{l}
u_{0} \\
v_{0} \\
\theta_{0}
\end{array}\right]=f_{0} \in Y,
$$

or, upon dividing by $\omega,|\omega| \geq 1$ :

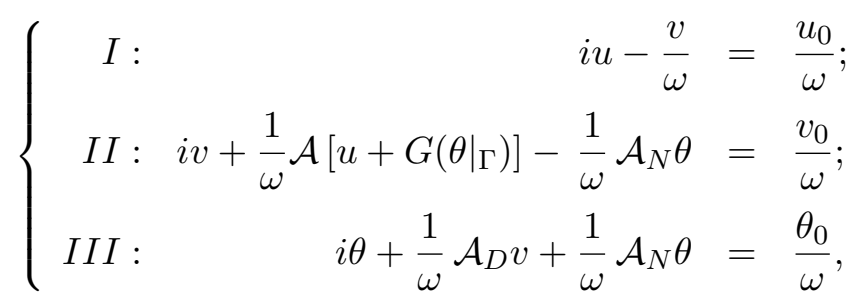

where, recalling (1.14) we have a-fortiori the following regularity properties

$$
y=[u, v, \theta] \in \mathcal{D}\left(\mathcal{A}_{D}\right) \times \mathcal{D}\left(\mathcal{A}_{D}\right) \times \mathcal{D}\left(\mathcal{A}_{N}\right),
$$

Orientation. The basic "driving" term in the present proof is the thermal estimate (2.2.3) below for $\theta$, which follows at once from the basic a-priori dissipativity condition (2.2.2). To achieve the desired estimates (2.1.4) through (2.1.6), we shall employ the "driving" estimate (2.2.3) repeatedly, along with a-priori bounds in the right norms, to dominate each norm-quantity $\|q\|$ of interest, as follows:

$$
\|q\| \leq[a+b]\left[\epsilon a+k_{\epsilon} b\right] \leq 2 \epsilon a^{2}+C_{\epsilon} b^{2}, a, b \geq 0,
$$

to be specialized with $a=\|y\|_{Y}$ and $b=\left\|\frac{f_{0}}{\omega}\right\|_{Y}$ [inequality (2.1.12) is obtained with $C_{\epsilon}=\left(\frac{k_{\epsilon}^{2}}{2 \epsilon}+k_{\epsilon}+\frac{\epsilon}{2}\right)$ by using $\epsilon a b \leq \frac{\epsilon}{2}\left(a^{2}+b^{2}\right)$ and $k_{\epsilon} a b \leq$ $\left.\frac{k_{\epsilon}}{2}\left(\frac{\epsilon}{k_{\epsilon}} a^{2}+\frac{k_{\epsilon}}{\epsilon} b^{2}\right)\right]$. 
2.2. A-priori bounds for $\theta, v$, and $u$. Part (i) of the following lemma is obtained by standard integration by parts, and is in fact behind the property of dissipativity of $A$ noted in Proposition 1.1(i). See [L-T.4, Chapter 3, Section 12] for details.

Lemma 2.2.1. (Preliminary a-priori bounds for $\theta$ ) With reference to (2.1.1), (2.1.2), we have with $\omega \in \mathbb{R}$ :

(i)

$$
\left(\mathcal{A}_{N} \theta, \theta\right)_{L_{2}(\Omega)}=\operatorname{Re}\left([i \omega I-A]\left[\begin{array}{l}
u \\
v \\
\theta
\end{array}\right],\left[\begin{array}{l}
u \\
v \\
\theta
\end{array}\right]\right)_{Y}=\operatorname{Re}\left(f_{0}, y\right)_{Y}
$$

(ii)

$$
\|\theta\|_{H^{1}(\Omega)}^{2} \doteq\left\|\mathcal{A}_{N}^{\frac{1}{2}} \theta\right\|_{L_{2}(\Omega)}^{2} \leq\left\|f_{0}\right\|_{Y}\|y\|_{Y}
$$

where here and henceforth, $\doteq$ denotes equivalence of norms;

(iii) for any $\epsilon>0$ and $\omega \in \mathbb{R}, \omega \neq 0$,

$$
\frac{1}{|\omega|}\|\theta\|_{H^{1}(\Omega)}^{2} \doteq \frac{1}{|\omega|}\left\|\mathcal{A}_{N}^{\frac{1}{2}} \theta\right\|_{L_{2}(\Omega)}^{2} \leq \frac{\epsilon}{2}\|y\|_{Y}^{2}+\frac{1}{2 \epsilon}\left\|\frac{f_{0}}{\omega}\right\|_{Y}^{2},
$$

Lemma 2.2.2 (A-priori bounds for $v$ ). With reference to (2.1.1) and (2.1.2), we have for $\omega \in \mathbb{R},|\omega| \geq 1$ :

(i)

$$
\begin{aligned}
\frac{1}{|\omega|}\left\|\mathcal{A}_{D} v\right\|_{L_{2}(\Omega)} \doteq \frac{1}{|\omega|}\|v\|_{H^{2}(\Omega)} & \leq\|u\|_{H^{2}(\Omega)}+\left\|\frac{u_{0}}{\omega}\right\|_{H^{2}(\Omega)} \\
& \leq\|y\|_{Y}+\left\|\frac{f_{0}}{\omega}\right\|_{Y}
\end{aligned}
$$

(ii)

$$
\frac{1}{\sqrt{|\omega|}}\left\|\mathcal{A}_{D}^{\frac{1}{2}} v\right\|_{L_{2}(\Omega)} \doteq \frac{1}{\sqrt{|\omega|}}\|v\|_{H^{1}(\Omega)} \leq C\left[\|y\|_{Y}+\left\|\frac{f_{0}}{\omega}\right\|_{Y}\right] .
$$

Proof. (i) The validity of estimate (2.2.4) stems at once from Eqn. I = (2.1.8), and the norm equivalence in (2.1.1). Then, (2.2.4) implies at once (2.2.5) by majorizing $u$ and $u_{0} / \omega$ in $H^{2}(\Omega)$ by $y$ and $f_{0} / \omega$ in $Y$, via (2.1.1) and (2.1.2).

(ii) By interpolation (moment inequality [L-M.1]), we compute [henceforth we use freely $\sqrt{a^{2}+b^{2}} \leq a+b$ for $a, b \geq 0$ throughout], via (2.2.5) and majorizing $v$ by $y$, by $(2.1 .1),(2.1 .2)$ :

$$
\|v\|_{H^{1}(\Omega)} \leq C\|v\|_{H^{2}(\Omega)}^{\frac{1}{2}}\|v\|_{L_{2}(\Omega)}^{\frac{1}{2}}
$$




$$
\begin{aligned}
(\text { by }(2.2 .5)) & \leq C|\omega|^{\frac{1}{2}}\left[\|y\|_{Y}^{\frac{1}{2}}+\left\|\frac{f_{0}}{\omega}\right\|^{\frac{1}{2}}\right]\|y\|_{Y}^{\frac{1}{2}} \\
& \leq C|\omega|^{\frac{1}{2}}\left[\|y\|_{Y}+\frac{1}{2}\left\|\frac{f_{0}}{\omega}\right\|+\frac{1}{2}\|y\|_{Y}\right],
\end{aligned}
$$

and (2.2.9) proves estimate (2.2.6), as desired.

Lemma 2.2.3 (Further a-priori bound for $\theta$ ). With reference to (2.1.1) and (2.1.2), we have for $\omega \in \mathbb{R},|\omega| \geq 1$ :

$$
\frac{1}{|\omega|}\|\theta\|_{H^{2}(\Omega)} \doteq \frac{1}{|\omega|}\left\|\mathcal{A}_{N} \theta\right\|_{L_{2}(\Omega)} \leq 2\left[\|y\|_{Y}+\left\|\frac{f_{0}}{\omega}\right\|_{Y}\right] \text {. }
$$

Proof. We return to Eqn. III = (2.1.10), where we use estimate $(2.2 .5)$ for $v$,

$$
\begin{aligned}
\left\|\frac{1}{\omega} \mathcal{A}_{N} \theta\right\|_{L_{2}(\Omega)} & =\left\|\frac{\theta_{0}}{\omega}-i \theta-\frac{1}{\omega} \mathcal{A}_{D} v\right\|_{L_{2}(\Omega)} \\
& \leq\left\|\frac{\theta_{0}}{\omega}\right\|_{L_{2}(\Omega)}+\|\theta\|_{L_{2}(\Omega)}+\frac{1}{|\omega|}\left\|\mathcal{A}_{D} v\right\|_{L_{2}(\Omega)} \\
(\operatorname{by}(2.2 .5)) & \leq\left\|\frac{f_{0}}{\omega}\right\|_{Y}+\|y\|_{Y}+\left[\|y\|_{Y}+\left\|\frac{f_{0}}{\omega}\right\|_{Y}\right],
\end{aligned}
$$

majorizing, in the last step, $\theta_{0}$ and $\theta$ by $f_{0}$ and $y$ via (2.1.1), (2.1.2). Then, (2.2.13) proves $(2.2 .10)$.

Lemma 2.2.4 (a-priori bounds for $u$ ). With reference to (2.1.1) and (2.1.2), we have for $\omega \in \mathbb{R},|\omega| \geq 1$ :

(i)

$$
\frac{1}{|\omega|}\|u\|_{H^{4}(\Omega)} \leq C\left[\|y\|_{Y}+\left\|\frac{f_{0}}{\omega}\right\|_{Y}\right]
$$

(ii)

$$
\frac{1}{\sqrt{|\omega|}}\|u\|_{H^{3}(\Omega)} \leq C\left[\|y\|_{Y}+\left\|\frac{f_{0}}{\omega}\right\|_{Y}\right]
$$

Proof. (i) Eqn. II $=(2.1 .9)$ rewritten abstractly as

$$
\frac{1}{\omega} \mathcal{A}\left[u+G\left(\left.\theta\right|_{\Gamma}\right)\right]=-i v+\frac{1}{\omega} \mathcal{A}_{N} \theta+\frac{v_{0}}{\omega}
$$

is equivalent, via the definition (1.7) of the Green operator $G$, to the following elliptic boundary value problem (i.e., the original elliptic problem (1.7)), of which (2.2.16) is the abstract version, in the first place):

$$
\begin{cases}\Delta^{2}\left(\frac{u}{\omega}\right)=-i v+\frac{1}{\omega} \mathcal{A}_{N} \theta+\frac{v_{0}}{\omega} & \text { in } \Omega \\ \left.u\right|_{\Gamma} \equiv 0 ;\left[\Delta u+\left.(1-\mu) B_{1} u\right|_{\Gamma}\right]=-\left.\theta\right|_{\Gamma} & \text { on } \Gamma .\end{cases}
$$


From the right-hand side of (2.2.17), we readily estimate, by virtue of $(2.2 .10)$ for $\mathcal{A}_{N} \theta / \omega$, majorizing $v$ and $\theta_{0}$ by $y$ and $f_{0}$ via (2.1.1), (2.1.2),

$$
\left\|\Delta^{2}\left(\frac{u}{\omega}\right)\right\|_{L_{2}(\Omega)} \leq 3\left[\|y\|_{Y}+\left\|\frac{f_{0}}{\omega}\right\|_{Y}\right] .
$$

Moreover, from the second B.C. in (2.2.18), we readily estimate for $|\omega| \geq 1$, by virtue of trace theory, (1.1f) for $B_{1}$, and again (2.2.10),

$$
\begin{aligned}
\left\|\Delta\left(\frac{u}{\omega}\right)\right\|_{H^{\frac{3}{2}(\Gamma)}} & \leq \frac{C}{|\omega|}\|u\|_{H^{2}(\Omega)}+\left\|\frac{\theta}{\omega}\right\|_{H^{\frac{3}{2}}(\Gamma)} \\
& \leq C\left[\|u\|_{H^{2}(\Omega)}+\frac{1}{|\omega|}\|\theta\|_{H^{2}(\Omega)}\right] \\
(\operatorname{by}(2.2 .10)) & \leq C\left[\|y\|_{Y}+\left\|\frac{f_{0}}{\omega}\right\|_{Y}\right]
\end{aligned}
$$

majorizing $u$ in $H^{2}(\Omega)$ by $y$ in $Y$, via (2.1.1), (2.1.2). Thus, standard elliptic theory [L-M.1; p. 188-9] applied to the 'right-hand side' estimate (2.2.19), the first B.C. in (2.2.18) and the boundary estimate (2.2.21), produces a gain of $2 \frac{1}{2}$ Sobolev units from boundary to interior $\left(\frac{3}{2}+2 \frac{1}{2}=4\right)$, and a gain of 4 Sobolev units from 'right-hand side' to interior $(0+4=4)$, thus yielding

$$
\left\|\frac{u}{\omega}\right\|_{H^{4}(\Omega)} \leq C\left[\|y\|_{Y}+\left\|\frac{f_{0}}{\omega}\right\|_{Y}\right],
$$

which proves $(2.2 .14)$. [We note that 'right-hand side' and boundary estimates produce, independently, the same interior regularity of $\frac{u}{\omega}$ in $H^{4}(\Omega)$.]

(ii) By interpolation (moment inequality), we estimate via (2.2.14), and majorizing $u$ by $y$, by (2.1.1), (2.1.2):

$$
\begin{aligned}
\|u\|_{H^{3}(\Omega)} & \leq C\|u\|_{H^{4}(\Omega)}^{\frac{1}{2}}\|u\|_{H^{2}(\Omega)}^{\frac{1}{2}} \\
& \leq C|\omega|^{\frac{1}{2}}\left[\|y\|_{Y}^{\frac{1}{2}}+\left\|\frac{f_{0}}{\omega}\right\|_{Y}^{\frac{1}{2}}\right]\|y\|_{Y}^{\frac{1}{2}} \\
& \leq C|\omega|^{\frac{1}{2}}\left[\|y\|_{Y}+\frac{1}{2}\left\|\frac{f_{0}}{\omega}\right\|_{Y}+\frac{1}{2}\|y\|_{Y}\right],
\end{aligned}
$$

and (2.2.24) proves estimate (2.2.15), as desired.

Remark 2.2.1. One could, alternatively, sum up Eqns. II $=(2.1 .9)$ and Eqn. III $=(2.1 .10)$ to eliminate $\left[\mathcal{A}_{N} \theta / \omega\right]$, and then use estimate $(2.2 .5)$ for $\left[\mathcal{A}_{D} v / w\right]$ (rather than estimate $(2.2 .10)$ on $\left[\mathcal{A}_{N} \theta / \omega\right]$, which is a consequence of (2.2.5), to obtain likewise the interior bound (2.2.19). It is, however, at the level of obtaining the boundary estimate (2.2.21) that estimate $(2.2 .10)$ is needed. 
2.3. A fundamental estimate on $\frac{1}{\omega}\left(\mathcal{A}_{D} v, \theta\right)_{L_{2}(\Omega)}$. The following resulta consequence of the 'driving' term (2.2.3) - is fundamental.

Proposition 2.3.1. With reference to (2.1.1), (2.1.2), given $\epsilon>0$ there exists $C_{\epsilon}>0$, such that for all $\omega \in \mathbb{R},|\omega| \geq 1$, we have

$$
\left|\frac{1}{\omega}\left(\mathcal{A}_{D} v, \theta\right)_{L_{2}(\Omega)}\right| \leq \epsilon\|y\|_{Y}^{2}+C_{\epsilon}\left\|\frac{f_{0}}{\omega}\right\|_{Y}^{2} .
$$

Proof. Step 1. By Green's first theorem with $v \in \mathcal{D}\left(\mathcal{A}_{D}\right)=H^{2}(\Omega) \cap$ $H_{0}^{1}(\Omega)$ and $\theta \in \mathcal{D}\left(\mathcal{A}_{N}\right)$, we have

$$
\begin{aligned}
-\frac{1}{\omega}\left(\mathcal{A}_{D} v, \theta\right)_{L_{2}(\Omega)} & =\frac{1}{\omega} \int_{\Omega} \Delta v \bar{\theta} d \Omega \\
& =\frac{1}{\omega} \int_{\Gamma} \frac{\partial v}{\partial \nu} \bar{\theta} d \Gamma-\frac{1}{\omega} \int_{\Omega} \nabla v \cdot \nabla \bar{\theta} d \Omega .
\end{aligned}
$$

Step 2. Lemma 2.3.2. In the same notation of Proposition 2.3.1, we have

(i)

$$
\left|\frac{1}{\omega} \int_{\Omega} \nabla v \cdot \nabla \bar{\theta} d \Omega\right| \leq \epsilon\|y\|_{Y}^{2}+C_{\epsilon}\left\|\frac{f_{0}}{\omega}\right\|_{Y}^{2}
$$

(ii)

$$
\left|\frac{1}{\omega} \int_{\Gamma} \frac{\partial v}{\partial \nu} \bar{\theta} d \Gamma\right| \leq \epsilon\|y\|_{Y}^{2}+C_{\epsilon}\left\|\frac{f_{0}}{\omega}\right\|_{Y}^{2}
$$

Assuming for the moment the validity of Lemma 2.3.2, we see that the desired conclusion (2.3.1) follows by use of estimates (2.3.3) and (2.3.4) in identity (2.3.2).

To prove Lemma 2.3.2, we shall use, for each part, inequality (2.1.12) plus a-priori bounds.

Step 3. Proof of inequality (2.3.3). By the a-priori bound (2.2.6) and the 'driving' bound (2.2.3), we estimate

$$
\text { 5) }\left|\frac{1}{\omega} \int_{\Omega} \nabla v \cdot \nabla \bar{\theta} d \Omega\right| \leq\left(\frac{1}{\sqrt{|\omega|}}\|v\|_{H^{1}(\Omega)}\right)\left(\frac{1}{\sqrt{|\omega|}}\|\theta\|_{H^{1}(\Omega)}\right)
$$

(by $(2.2 .6)$ and $(2.2 .3)) \leq C\left[\|y\|_{Y}+\left\|\frac{f_{0}}{\omega}\right\|_{Y}\right]\left[\sqrt{\epsilon_{1}}\|y\|_{Y}+\frac{1}{\sqrt{\epsilon_{1}}}\left\|\frac{f_{0}}{\omega}\right\|_{Y}\right]$

$$
(\text { by }(2.1 .12)) \leq C \sqrt{\epsilon_{1}}\|y\|_{Y}^{2}+C_{\epsilon_{1}}\left\|\frac{f_{0}}{\omega}\right\|_{Y}^{2},
$$


Step 4. Proof of inequality (2.3.4). We recall the a-priori inequalities [Th.1], [B-S.1, p. 37]

$$
\begin{aligned}
\left\|\frac{\partial v}{\partial \nu}\right\|_{L_{2}(\Gamma)} & \leq C\|v\|_{H^{2}(\Omega)}^{\frac{1}{2}}\|v\|_{H^{1}(\Omega)}^{\frac{1}{2}} \\
\left\|\left.\theta\right|_{\Gamma}\right\|_{L_{2}(\Gamma)} & \leq C\|\theta\|_{H^{1}(\Omega)}^{\frac{1}{2}}\|\theta\|_{L_{2}(\Omega)}^{\frac{1}{2}} .
\end{aligned}
$$

Then, using (2.3.8) and (2.3.9),

$$
\begin{aligned}
\left|\frac{1}{\omega} \int_{\Gamma} \frac{\partial v}{\partial \nu} \bar{\theta} d \Gamma\right| & \leq \frac{1}{|\omega|}\left\|\left.\frac{\partial v}{\partial \nu}\right|_{\Gamma}\right\|_{L_{2}(\Gamma)}\left\|\left.\theta\right|_{\Gamma}\right\|_{L_{2}(\Gamma)} \\
& \leq C \frac{\|v\|_{H^{2}(\Omega)}^{\frac{1}{2}}}{\sqrt{|\omega|}} \frac{\|v\|_{H^{1}(\Omega)}^{\frac{1}{2}}}{|\omega|^{\frac{1}{4}}} \frac{\|\theta\|_{H^{1}(\Omega)}^{\frac{1}{2}}}{|\omega|^{\frac{1}{4}}}\|\theta\|_{L_{2}(\Omega)}^{\frac{1}{2}} .
\end{aligned}
$$

Taking the $\frac{1}{2}$-th power of the a-priori bounds (2.2.5) and (2.2.6), we obtain the following uniform bound for $|\omega| \geq 1$ :

$$
\begin{aligned}
\frac{\|v\|_{H^{2}(\Omega)}^{\frac{1}{2}}}{\sqrt{|\omega|}} \frac{\|v\|_{H^{1}(\Omega)}^{\frac{1}{2}}}{|\omega|^{\frac{1}{4}}} & \leq C\left[\|y\|_{Y}^{\frac{1}{2}}+\left\|\frac{f_{0}}{\omega}\right\|_{Y}^{\frac{1}{2}}\right]\left[\|y\|_{Y}^{\frac{1}{2}}+\left\|\frac{f_{0}}{\omega}\right\|_{Y}^{\frac{1}{2}}\right] \\
& \leq C\left[\|y\|_{Y}+\left\|\frac{f_{0}}{\omega}\right\|_{Y}\right] .
\end{aligned}
$$

On the other hand, taking the $\frac{1}{4}$-power of the 'driving' bound (2.2.3) and majorizing $\theta$ by $y$ by (2.1.1), (2.1.2), we obtain the following uniform bound for $|\omega| \geq 1$,

$$
\begin{aligned}
\frac{\|\theta\|_{H^{1}(\Omega)}^{\frac{1}{2}}}{|\omega|^{\frac{1}{4}}}\|\theta\|_{L_{2}(\Omega)}^{\frac{1}{2}} & \leq 2^{-\frac{1}{4}}\left[\epsilon_{1}\|y\|_{Y}^{\frac{1}{2}}+\frac{1}{\epsilon_{1}}\left\|\frac{f_{0}}{\omega}\right\|_{Y}^{\frac{1}{2}}\right]\|y\|_{Y}^{\frac{1}{2}} \\
& \leq 2^{-\frac{5}{4}}\left[3 \epsilon_{1}\|y\|_{Y}+\frac{1}{\epsilon_{1}^{3}}\left\|\frac{f_{0}}{\omega}\right\|_{Y}\right] .
\end{aligned}
$$

Using estimates (2.3.12) and (2.3.14) on the right-hand side of (2.3.10), we obtain

(2.3.15) $\left|\frac{1}{\omega} \int_{\Gamma} \frac{\partial v}{\partial \nu} \bar{\theta} d \Gamma\right| \leq C\left[\|y\|_{Y}+\left\|\frac{f_{0}}{\omega}\right\|_{Y}\right]\left[\epsilon_{1}\|y\|_{Y}+\frac{1}{\epsilon_{1}^{3}}\left\|\frac{f_{0}}{\omega}\right\|_{Y}\right]$

$$
(\text { by }(2.1 .12)) \leq C\left[2 \epsilon_{1}\|y\|_{Y}^{2}+C_{\epsilon_{1}}\left\|\frac{f_{0}}{\omega}\right\|_{Y}^{2}\right] \text {, }
$$

recalling (2.1.12) in the last step, $\epsilon_{1}>0$ being arbitrary. Then, inequality (2.3.16) proves (2.3.4), as desired. The proof of Lemma 2.3.2 is complete, and so is the proof of Proposition 2.3.1.

2.4. Proof of estimate $\mathbf{( 2 . 1 . 6 )}$ for $\theta$. As a corollary of the 'driving' estimate (2.2.3) for $\theta$, as well as of Proposition 2.3.1 (which also stems from (2.2.3), we obtain the desired inequality (2.1.6) for $\theta$. 
Proposition 2.4.1. With reference to (2.1.1), (2.1.2), given $\epsilon>0$ there exists $C_{\epsilon}>0$ such that for all $\omega \in \mathbb{R},|\omega| \geq 1$, we have

$$
\|\theta\|_{L_{2}(\Omega)}^{2} \leq \epsilon\|y\|_{Y}^{2}+C_{\epsilon}\left\|\frac{f_{0}}{\omega}\right\|_{Y}^{2}
$$

Proof. We return to Eqn. III $=(2.1 .10)$, take here the $L_{2}(\Omega)$-inner product with $\theta$, use estimates (2.3.1) and (2.2.3), and obtain

$$
\begin{aligned}
\|\theta\|_{L_{2}(\Omega)}^{2} \leq & \left|\frac{1}{\omega}\left(\mathcal{A}_{D} v, \theta\right)_{L_{2}(\Omega)}\right| \\
& +\frac{1}{|\omega|}\left\|\mathcal{A}_{N}^{\frac{1}{2}} \theta\right\|_{L_{2}(\Omega)}^{2}+\left|\left(\frac{\theta_{0}}{\omega}, \theta\right)_{L_{2}(\Omega)}\right|
\end{aligned}
$$

(by $(2.3 .1)$ and $(2.2 .3)) \leq\left[\epsilon\|y\|_{Y}^{2}+C_{\epsilon}\left\|\frac{f_{0}}{\omega}\right\|_{Y}^{2}\right]+\frac{1}{2}\left[\epsilon\|y\|_{Y}^{2}+\frac{1}{\epsilon}\left\|\frac{f_{0}}{\omega}\right\|_{Y}^{2}\right]$

$$
+\frac{\epsilon}{2}\|\theta\|_{L_{2}(\Omega)}^{2}+\frac{1}{2 \epsilon}\left\|\frac{\theta_{0}}{\omega}\right\|_{Y}^{2} \text {. }
$$

Then, the desired inequality (2.4.1) readily follows from (2.4.3), by majorizing $\theta_{0}$ by $f_{0}$ and $\theta$ by $y$, via $(2.1 .1),(2.1 .2)$.

2.5. Improving upon a-priori bounds. The 'driving' estimate (2.2.3) and the a-priori bound $(2.2 .6)$ for $\left[\mathcal{A}_{D}^{\frac{1}{2}} v / \sqrt{|\omega|}\right]$ yield

Lemma 2.5.1. With reference to (2.1.1), (2.1.2), given $\epsilon>0$ there exists $C_{\epsilon}>0$ such that for all $\omega \in \mathbb{R},|\omega| \geq 1$,

$$
\left|\frac{1}{\omega}\left(\mathcal{A}_{N} \theta, v\right)_{L_{2}(\Omega)}\right| \leq \epsilon\|y\|_{Y}^{2}+C_{\epsilon}\left\|\frac{f_{0}}{\omega}\right\|_{Y}^{2} .
$$

Proof. With $\theta \in \mathcal{D}\left(\mathcal{A}_{N}\right)$ and $v \in \mathcal{D}\left(\mathcal{A}_{D}\right) \subset H^{1}(\Omega)=\mathcal{D}\left(\mathcal{A}_{N}^{\frac{1}{2}}\right)$, we estimate by $(2.2 .3)$ and (2.2.6):

$$
\begin{aligned}
\left|\frac{1}{\omega}\left(\mathcal{A}_{N} \theta, v\right)_{L_{2}(\Omega)}\right| & =\left|\left(\frac{\mathcal{A}_{N}^{\frac{1}{2}} \theta}{\sqrt{|\omega|}}, \frac{\mathcal{A}_{N}^{\frac{1}{2}} v}{\sqrt{|\omega|}}\right)_{L_{2}(\Omega)}\right| \\
& \leq \frac{\left\|\mathcal{A}_{N}^{\frac{1}{2}} \theta\right\|_{L_{2}(\Omega)}}{\sqrt{|\omega|}} \frac{\left\|\mathcal{A}_{N}^{\frac{1}{2}} v\right\|_{L_{2}(\Omega)}}{\sqrt{|\omega|}} \\
(\operatorname{by}(2.2 .3),(2.2 .6)) & \leq C\left[\epsilon_{1}\|y\|_{Y}+\frac{1}{\epsilon_{1}}\left\|\frac{f_{0}}{\omega}\right\|_{Y}\right]\left[\|y\|_{Y}+\left\|\frac{f_{0}}{\omega}\right\|_{Y}\right]
\end{aligned}
$$




$$
\text { (by }(2.1 .12)) \leq C \epsilon_{1}\|y\|_{Y}^{2}+C_{\epsilon_{1}}\left\|\frac{f_{0}}{\omega}\right\|_{Y}^{2},
$$

after invoking (2.1.12) in the last step, for an arbitrary $\epsilon_{1}>0$. Then, (2.5.4) proves (2.5.1), as desired.

The following result - a corollary of Proposition 2.4.1, Eqn. (2.4.1), for $\theta$-improves upon the a-priori bound (2.2.6) for $v$.

Lemma 2.5.2. With reference to (2.1.1), (2.1.2), given $\epsilon>0$ there exists $C_{\epsilon}>0$ such that for all $\omega \in \mathbb{R},|\omega| \geq 1$, we have

$$
\frac{1}{|\omega|}\|v\|_{H^{1}(\Omega)}^{2} \doteq \frac{1}{|\omega|}\left\|\mathcal{A}_{D}^{\frac{1}{2}} v\right\|_{L_{2}(\Omega)}^{2} \leq \epsilon\|y\|_{Y}^{2}+C_{\epsilon}\left\|\frac{f_{0}}{\omega}\right\|_{Y}^{2} .
$$

Proof. We return to Eqn. III $=(2.1 .10)$, take here the $L_{2}(\Omega)$-inner product with $v$, invoke estimates (2.4.1) and (2.5.1), thereby obtaining

$$
\begin{aligned}
\frac{1}{|\omega|}\left\|\mathcal{A}_{D}^{\frac{1}{2}} v\right\|_{L_{2}(\Omega)}^{2}= & \left|\frac{1}{\omega}\left(\mathcal{A}_{D} v, v\right)_{L_{2}(\Omega)}\right| \\
= & \left|\left(\frac{\theta_{0}}{\omega}-i \theta-\frac{1}{\omega} \mathcal{A}_{N} \theta, v\right)_{L_{2}(\Omega)}\right| \\
\leq & \left\|\frac{\theta_{0}}{\omega}\right\|_{L_{2}(\Omega)}\|v\|_{L_{2}(\Omega)}+\|\theta\|_{L_{2}(\Omega)}\|v\|_{L_{2}(\Omega)} \\
& +\left|\frac{1}{\omega}\left(\mathcal{A}_{N} \theta, v\right)_{L_{2}(\Omega)}\right|
\end{aligned}
$$

$$
\begin{aligned}
(\text { by }(2.4 .1),(2.5 .1)) \leq & {\left[\frac{\epsilon_{1}}{2}\|v\|_{L_{2}(\Omega)}^{2}+\frac{1}{2 \epsilon_{1}}\left\|\frac{\theta_{0}}{\omega}\right\|_{L_{2}(\Omega)}^{2}\right] } \\
& +\left[\epsilon_{1}\|y\|_{Y}+C_{\epsilon_{1}}\left\|\frac{f_{0}}{\omega}\right\|_{Y}\right]\|y\|_{Y} \\
& +\left[\epsilon_{1}\|y\|_{Y}^{2}+C_{\epsilon_{1}}\left\|\frac{f_{0}}{\omega}\right\|_{Y}^{2}\right] \\
\leq & \epsilon\|y\|_{Y}^{2}+C_{\epsilon}\left\|\frac{f_{0}}{\omega}\right\|_{Y}^{2}
\end{aligned}
$$

majorizing $v$ by $y$ twice via (2.1.1), (2.1.2), once from (2.5.7) to (2.5.8), and once from (2.5.8) to (2.5.9). Eqn. (2.5.9) proves (2.5.5).

2.6. Proof of estimate (2.1.5) for $v$. As a corollary of Lemma 2.5.2 and of the a-priori bound Eqn. (2.2.15), Lemma 2.2.4 on $u$, we obtain the desired estimate (2.1.5) for $v$. 
Proposition 2.6.1. With reference to (2.1.1), (2.1.2), given $\epsilon>0$ there exists $C_{\epsilon}>0$ such that for all $\omega \in \mathbb{R},|\omega| \geq 1$ :

$$
\|v\|_{L_{2}(\Omega)}^{2} \leq \epsilon\|y\|_{Y}^{2}+C_{\epsilon}\left\|\frac{f_{0}}{\omega}\right\|_{Y}^{2} .
$$

Proof. Step 1. We return to Eqn. II $=(2.1 .9)$ and take here the $L_{2}(\Omega)$ inner product with $v$, thereby obtaining, see definitions (1.2) and (1.7) of the operators $\mathcal{A}$ and $G$ :

$$
\|v\|_{L_{2}(\Omega)}^{2} \leq\left|\frac{1}{\omega} \int_{\Omega} \Delta^{2} u \bar{v} d \Omega\right|+\left|\frac{1}{\omega}\left(\mathcal{A}_{N} \theta, v\right)_{L_{2}(\Omega)}\right|+\left|\left(\frac{\theta_{0}}{\omega}, v\right)_{L_{2}(\Omega)}\right| .
$$

Step 2. Lemma 2.6.2. In the notation of Proposition 2.6.1, we have

$$
\left|\frac{1}{\omega} \int_{\Omega} \Delta^{2} u \bar{v} d \Omega\right| \leq \epsilon\|y\|_{Y}^{2}+C_{\epsilon}\left\|\frac{f_{0}}{\omega}\right\|_{Y}^{2} .
$$

Proof of Lemma 2.6.2. Since $v \in \mathcal{D}\left(\mathcal{A}_{D}\right)=H^{2}(\Omega) \cap H_{0}^{1}(\Omega)$, then $\left.\bar{v}\right|_{\Gamma}=0$ and Green's first theorem yields by virtue of (2.2.15) and (2.5.5),

$$
\begin{aligned}
\left|\frac{1}{\omega} \int_{\Omega} \Delta^{2} u \bar{v} d \Omega\right| & =\left|\frac{1}{\omega} \int \nabla \Delta u \cdot \nabla \bar{v} d \Omega\right| \\
& \leq\left(\frac{1}{\sqrt{|\omega|}}\|u\|_{H^{3}(\Omega)}\right)\left(\frac{1}{\sqrt{|\omega|}}\|v\|_{H^{1}(\Omega)}\right)
\end{aligned}
$$

(2.6.6) $($ by $(2.2 .15),(2.5 .5)) \leq C\left[\|y\|_{Y}+\left\|\frac{f_{0}}{\omega}\right\|_{Y}\right]\left[\epsilon_{1}\|y\|_{Y}+C_{\epsilon_{1}}\left\|\frac{f_{0}}{\omega}\right\|_{Y}\right]$

$$
(\operatorname{by}(2.1 .12)) \leq C\left[\epsilon_{1}\|y\|_{Y}^{2}+C_{\epsilon_{1}}\left\|\frac{f_{0}}{\omega}\right\|_{Y}^{2}\right],
$$

invoking (2.1.12) in the last step, with $\epsilon_{1}>0$ arbitrary. Then (2.6.7) proves (2.6.3).

Step 3. We use estimate (2.6.3) and estimate (2.5.1) on the right-hand side of (2.6.2) to obtain

$$
\begin{aligned}
\|v\|_{L_{2}(\Omega)}^{2} \leq & 2\left[\epsilon_{1}\|y\|_{Y}^{2}+C_{\epsilon_{1}}\left\|\frac{f_{0}}{\omega}\right\|_{Y}^{2}\right] \\
& +\left[\frac{\epsilon_{1}}{2}\|v\|_{L_{2}(\Omega)}^{2}+\frac{1}{2 \epsilon_{1}}\left\|\frac{\theta_{0}}{\omega}\right\|_{L_{2}(\Omega)}^{2}\right],
\end{aligned}
$$

from which the desired estimate (2.6.1) follows at once, majorizing $v$ and $\theta_{0}$ by $y$ and $f_{0}$, via $(2.1 .1),(2.1 .2)$. 


\subsection{Proof of estimate (2.1.4) for $u$.}

Proposition 2.7.1. With reference to (2.1.1), (2.1.2), given $\epsilon>0$, there is $C_{\epsilon}>0$ such that for all $\omega \in \mathbb{R},|\omega| \geq 1$,

$$
\|u\|_{H^{2}(\Omega)}^{2} \leq \epsilon\|y\|_{Y}^{2}+C_{\epsilon}\left\|\frac{f_{0}}{\omega}\right\|_{Y}^{2} .
$$

Proof. Step 1. We have already noted in Lemma 2.6.2, recalling the definitions (1.2) and (1.7) of the operators $\mathcal{A}$ and $G$, that

$$
\begin{aligned}
\left|\frac{1}{\omega}\left(\mathcal{A}\left[u+G\left(\left.\theta\right|_{\Gamma}\right)\right], v\right)_{L_{2}(\Omega)}\right| & =\left|\frac{1}{\omega} \int_{\Omega} \Delta^{2} u \bar{v} d \Omega\right| \\
& \leq \epsilon_{1}\|y\|_{Y}^{2}+C_{\epsilon_{1}}\left\|\frac{f_{0}}{\omega}\right\|_{Y}^{2} .
\end{aligned}
$$

Step 2. On the other hand, by substituting $\frac{v}{w}=i u-\frac{u_{0}}{\omega}$ from Eqn. I $=$ (2.1.8), we obtain

$(2.7 .3) \frac{1}{\omega}\left(\mathcal{A}\left[u+G\left(\left.\theta\right|_{\Gamma}\right)\right], v\right)_{L_{2}(\Omega)}=\left(\mathcal{A} u, i u-\frac{u_{0}}{\omega}\right)_{L_{2}(\Omega)}$

$$
+\frac{1}{\omega}\left(\left.\theta\right|_{\Gamma}, G^{*} \mathcal{A} v\right)_{L_{2}(\Gamma)}
$$

$$
\begin{aligned}
(\operatorname{by}(1.9))= & -i\left\|\mathcal{A}^{\frac{1}{2}} u\right\|_{L_{2}(\Omega)}^{2}-\left(\mathcal{A}^{\frac{1}{2}} u, \frac{\mathcal{A}^{\frac{1}{2}} u_{0}}{\omega}\right)_{L_{2}(\Omega)} \\
& +\frac{1}{\omega}\left(\left.\theta\right|_{\Gamma},\left.\frac{\partial v}{\partial \nu}\right|_{\Gamma}\right)_{L_{2}(\Gamma)},
\end{aligned}
$$

recalling $G^{*} \mathcal{A} v=\frac{\partial v}{\partial \nu}$ from (1.9) in the last step.

Step 3. Combining (2.7.3) with (2.7.2), we obtain via the norm equivalence in (1.5):

$$
\begin{aligned}
\|u\|_{H^{2}(\Omega)}^{2} \doteq & \left\|\mathcal{A}^{\frac{1}{2}} u\right\|_{L_{2}(\Omega)}^{2} \leq\left[\epsilon_{1}\|y\|_{Y}^{2}+C_{\epsilon_{1}}\left\|\frac{f_{0}}{\omega}\right\|_{Y}^{2}\right] \\
& +\left[\frac{\epsilon_{1}}{2}\left\|\mathcal{A}^{\frac{1}{2}} u\right\|_{L_{2}(\Omega)}^{2}+\frac{1}{2 \epsilon_{1}}\left\|\frac{\mathcal{A}^{\frac{1}{2}} u_{0}}{\omega}\right\|_{Y}^{2}\right] \\
& +\left|\frac{1}{\omega}\left(\left.\theta\right|_{\Gamma},\left.\frac{\partial v}{\partial \nu}\right|_{\Gamma}\right)_{L_{2}(\Gamma)}\right|
\end{aligned}
$$




$$
(\text { by }(2.3 .4)) \leq \epsilon_{2}\|y\|_{Y}^{2}+C_{\epsilon_{2}}\left\|\frac{f_{0}}{\omega}\right\|_{Y}^{2}+\frac{\epsilon_{1}}{2}\left\|\mathcal{A}^{\frac{1}{2}} u\right\|_{L_{2}(\Omega)}^{2},
$$

where in going from (2.7.5) to (2.7.6) we have invoked the boundary estimate (2.3.4) of Lemma 2.3.2, and have majorized $\mathcal{A}^{\frac{1}{2}} u_{0}$ by $f_{0}$ by (2.1.1). Estimate (2.7.6) readily leads to the desired conclusion (2.7.1).

The required estimates (2.1.4), (2.1.5), (2.1.6) are all proved.

Final Remark. Having shown the required estimate (2.7.1) for $u$, we return to Eqn. $\mathrm{I}=(2.1 .8)$ and obtain: given $\epsilon>0$ there exists $C_{\epsilon}>0$ such that for all $\omega \in \mathbb{R},|\omega| \geq 1$, we have

$$
\frac{1}{|\omega|}\left\|\mathcal{A}_{D} v\right\|_{L_{2}(\Omega)} \doteq \frac{1}{|\omega|}\|v\|_{H^{2}(\Omega)} \leq \epsilon\|y\|_{Y}+C_{\epsilon}\left\|\frac{f_{0}}{\omega}\right\|_{Y} .
$$

Moreover, armed with estimate (2.7.7), we return to Eqn. III $=(2.1 .10)$, use here also estimate (2.4.1) for $\theta$, and obtain

$$
\begin{aligned}
\frac{1}{|\omega|}\|\theta\|_{H^{2}(\Omega)} \doteq \frac{1}{|\omega|}\left\|\mathcal{A}_{N} \theta\right\|_{L_{2}(\Omega)} & =\left\|\frac{\theta_{0}}{\omega}-i \theta-\frac{1}{\omega} \mathcal{A}_{D} v\right\|_{L_{2}(\Omega)} \\
(\operatorname{by~}(2.4 .1),(2.7 .7)) & \leq \epsilon\|y\|_{Y}+C_{\epsilon}\left\|\frac{f_{0}}{\omega}\right\|_{Y},
\end{aligned}
$$

majorizing also $\theta_{0}$ by $f_{0}$ via (2.1.1). Inequality (2.7.9) complements the 'driving' estimate (2.2.3) as well as its consequence (2.4.1).

\section{REFERENCES}

[B-S.1] S. C. Brenner and L. R. Scott, The Mathematical Theory of Finite Element Methods, Texts in Appl. Math., \#15, Springr-Verlag, New York, 1994.

[Cg-T.1] S. K. Chang and R. Triggiani, Spectral analysis of thermo-elastic plates with rotational forces, in "Optimal Control: Theory, Algorithms, Applications", (eds. W. W. Hager and P. M. Pardalos), Kluwer, 1998, 84-115.

[C-T.1] S. Chen and R. Triggiani, Proof of two conjectures of G. Chen and D. L. Russell on structural damping for elastic systems: The case $\alpha=\frac{1}{2}$. Lecture Notes in Math., \#1354, Springer-Verlag, New York, 1988; Proc. "Seminar in Approximation and Optimization", Havana, Cuba, January 12-14, 1987.

[C-T.2] S. Chen and R. Triggiani, Proof of extension of two conjectures on structural damping for elastic systems: The case $\frac{1}{2} \leq \alpha \leq 1$, Pacific J. Math. 136 (1989), $15-55$.

[G.1] P. Grisvard, Caractérisation de quelques espaces d'interpolation. Arch. Rational Mech. Anal. 25 (1967), 40-63.

[H-P-L.1] D. Henry, A. Perissinitto and O. Lopez, On the essential spectrum of a semigroup of thermo-elasticity, Nonlinear Anal. 21 (1993), 65-75.

[Kr.1] S. G. Krein, Linear Differential Equations in Banach space, Transl. Math. Monographs, \#29, Amer. Math. Soc., Providence, RI, 1988.

[Lag.1] J. Lagnese, Boundary Stabilization of Thin Plates, SIAM Studies in Appl. Math., \#10, Philadelphia, 1989.

[Las.1] I. Lasiecka, Control and stabilization of interactive structures, Systems and Control in the 21st Century, Birkhauser, (1997), 245-262.

[L-T.1] I. Lasiecka and R. Triggiani, Two direct proofs on the analiticity of the s.c. semigroup arising in abstract thermo-elastic equations, Advances Differential Equations, 3 (1998), 387-416. 
[L-T.2] I. Lasiecka and R. Triggiani, Structural decomposition of the s.c. semigroup arising in thermo-elastic plate theory with rotational forces, IFIP Workshop, Univ. Florida, February 1977, Semigroup Forum, to appear.

[L-T.3] I. Lasiecka and R. Triggiani, Analyticity and lack thereof, of thermo-elastic semigroups, ESAIM Proc., to appear.

[L-T.4] I. Lasiecka and R. Triggiani, Control Theory for Partial Differential Equations: Continuous and Approximation Theories, Vol. I, Cambridge Univ. Press, Encyclopedia of Mathematics and its Applications, to appear in 1998.

[L-T.5] I. Lasiecka and R. Triggiani, Analyticity of thermo-elastic semigroups with free B.C., Ann. Scuola Norm. Sup. Pisa Cl. Sci. (4), to appear. Presented at workshop on "Deterministic and stochastic evolution equations," Scuola Normale Superiore, Pisa, Italy, July 1997; IFIP Conference, Detroit, July 1997; MMAR'97, Miedzyzdroje, Poland, August 1997; Conference on Differential Equations, Vanderbilt U., November 1997.

[L-M.1] J. L. Lions and E. Magenes, Nonhomogeneous Boundary Value Problems and Applications I, Springer-Verlag, Berlin, 1972,

[L-L.1] K. Liu and Z. Liu, Exponential stability and analyticity of abstract linear thermo-elastic systems, Z. Agnew. Math. Phys. 48 (1997), 885-904.

[L-R.1] Z. Liu and M. Renardy, A note on the equations of thermoelastic plate, Appl. Math. Lett. 8 (1995), 1-6.

[P.1] A. Pazy, Semigroups of Linear Operators and Application to Partial Differential Equations, Springer-Verlag, Berlin, 1983.

[T.1] R. Triggiani, Analyticity, and lack thereof, of semigroups arising from thermoelastic plates, in "Proceedings of Computational Science for the 21st Century", John Wiley, May 5-7, 1997.

[T.2] R. Triggiani, Thermo-elastic equations of Euler-Bernoulli or Kirchoff type: Analyticity, or lack thereof, of the corresponding s.c. semigroup, in "Proceedings of Fourth International Symposium MMAR'97", \#1, (1997), 89-96.

[Th.1] V. Thomee, Galerkin Finite Element Methods for Parabolic Problems, Lecture Notes in Math., \#1054, Springer-Verlag, Berlin, 1984.

[T-Z.1] L. de Teresa and E. Zuazua, Controllability of the linear system of thermoelastic plates, Advances Differential Equations, 1 (1996), 369-402.

Department of Mathematics

KeRChOF HALL

UNIVERSITY OF VIRGINIA

Charlottesville, VA 22903, USA

E-mail address: il2v@virginia.edu,rt7u@amsun.apma.virginia.edu 


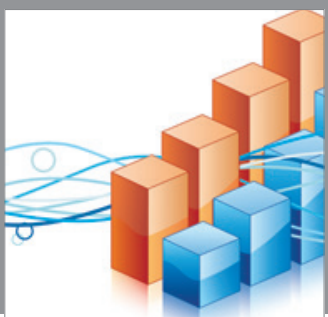

Advances in

Operations Research

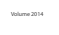

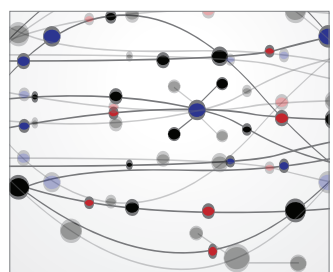

\section{The Scientific} World Journal
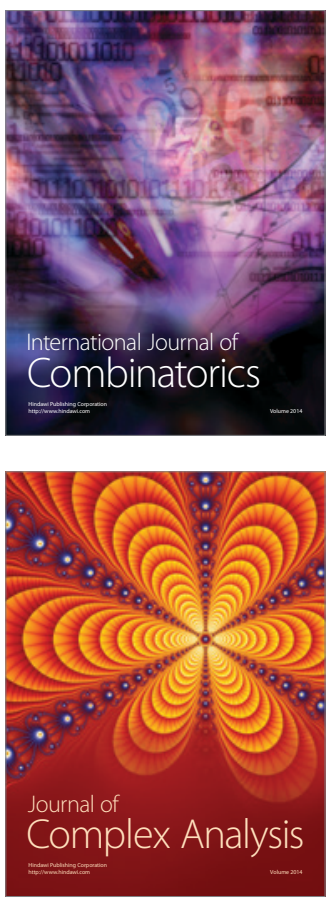

International Journal of

Mathematics and

Mathematical

Sciences
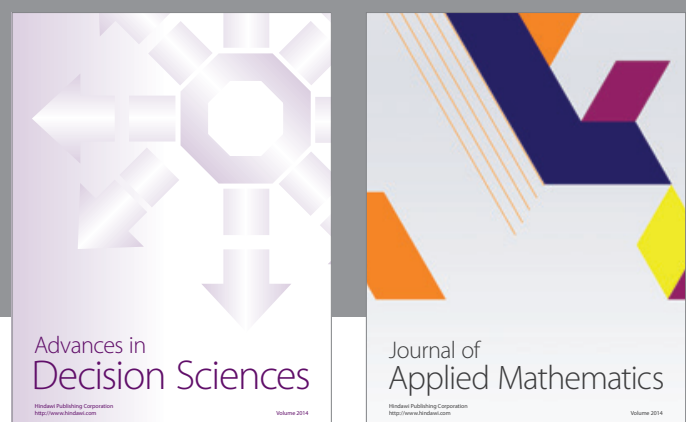

Journal of

Applied Mathematics
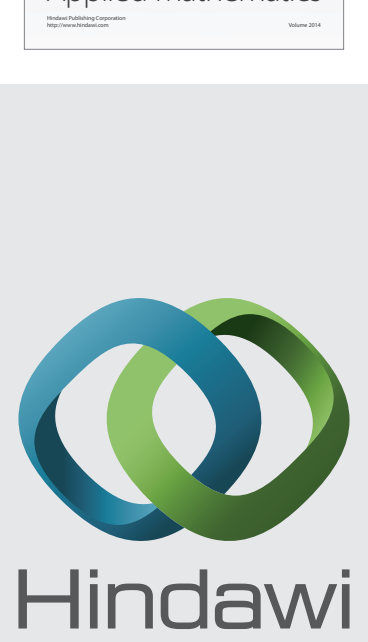

Submit your manuscripts at http://www.hindawi.com
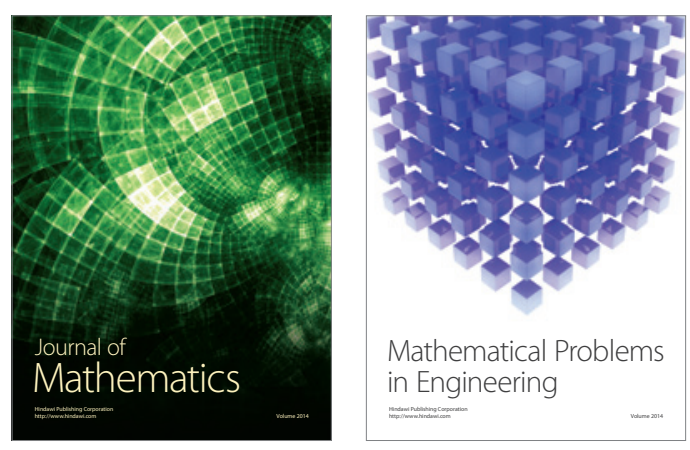

Mathematical Problems in Engineering
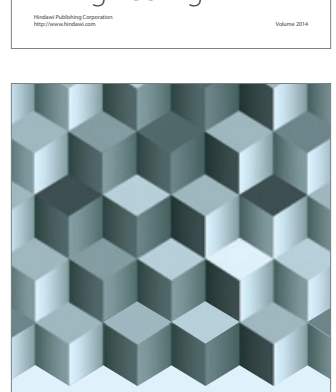

Journal of

Function Spaces
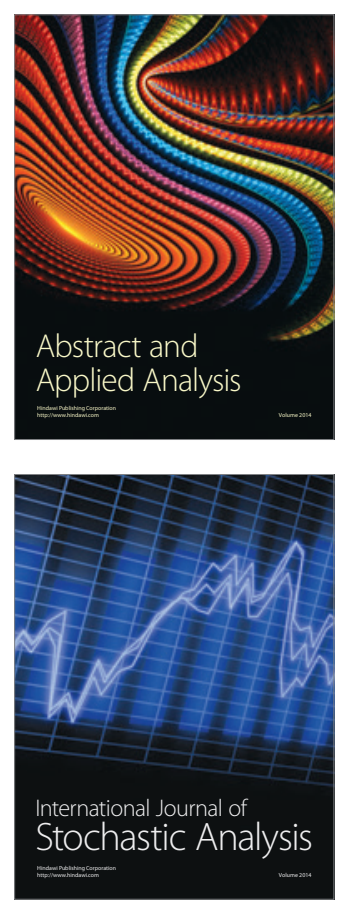

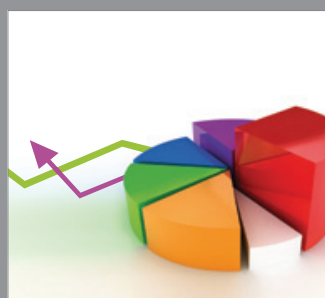

ournal of

Probability and Statistics

Promensencen
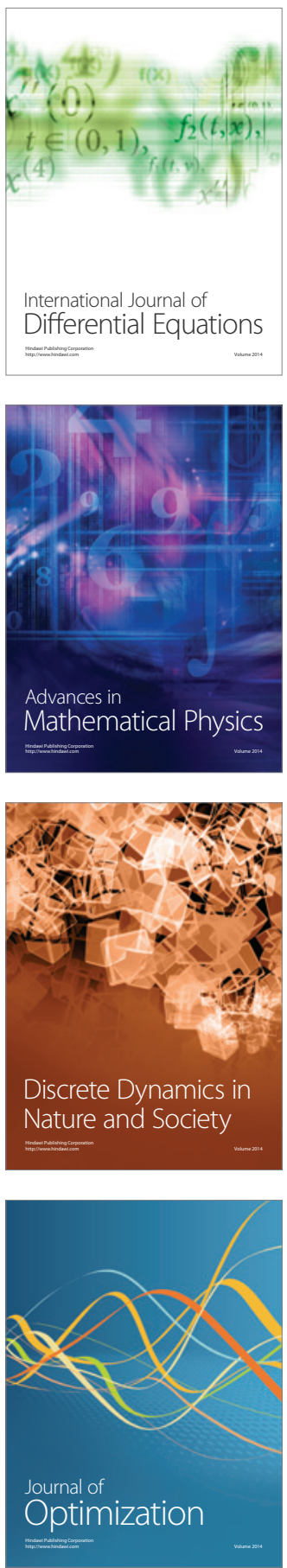